\title{
MECHANISMS FOR IDENTIFYING THE PATTERNS OF THE DYNAMICS OF SCIENTIFIC AND TECHNICAL PUBLICATIONS ON THE EXAMPLE OF THE THEMATIC DIRECTION «ROBOTICS»
}

\author{
M.V. Cherkasskaya ${ }^{1, a}$, A.I. Cherkasskiy ${ }^{2}$, L.V. Pronicheva ${ }^{2}$ \\ ${ }^{1}$ Plekhanov Russian University of Economics, 36 Stremyanny lane, Moscow, 117997, Russia \\ ${ }^{2}$ National Research Nuclear University MEPhI (Moscow Engineering Physics Institute), 31 \\ Kashirskoe hwy, Moscow, 115409, Russia \\ E-mail: ${ }^{a}$ CHerkasskaya.MV @ rea.ru
}

Scientific activity is a source of new knowledge and the creation of the latest technologies to improve the quality of life. The results of research are presented in the form of articles published in scientific journals or collections of scientific conferences, thereby being the main channel of communication in the scientific environment, and also characterize the state of the scientific organization and the country in the world scientific ranking. The article analyzes the world publication activity of countries on the example of the direction - "Robotics" based on the data of the Web of Science system. It was revealed that the development of technologies is subject to certain laws and it is possible to build predicative models for the emergence of new technologies.

Keywords: publication activity, robotics, Web of Science, predictive analysis

Marina Cherkasskaya, Andrey Cherkasskiy, Larisa Pronicheva

Copyright (C) 2021 for this paper by its authors. Use permitted under Creative Commons License Attribution 4.0 International (CC BY 4.0). 


\section{Introduction}

In world practice, the number of published articles in leading scientific publications is indicators of the results of scientific activities of researchers, research organizations and higher educational institutions. International publication activity reflects the level of development of national science against the background of other countries, especially in the field of basic research, where there can be no results other than publications by definition. In developed countries, to track and analyze the dynamics of scientific information flows, information and analytical systems have been developed that aggregate scientific publications [1]. Currently, the most famous systems of this type are Web of Science (WoS) and Scopus.

Both databases are widely used in many countries of the world to assess the effectiveness of both individual researchers and scientific teams and institutions. Using the built-in tools of information and analytical systems, it is possible to analyze the publication activity in any area of research of interest [2].

\section{Rationale for selecting a study topic}

Robotics is an applied science based on cybernetics, bionics and mechanics, engaged in the development of automated technical systems based on electronics and programming. Robotics studies both the theory, methods of calculating and designing robots, their systems and elements, and the problems of complex automation of production and scientific research using robots [3].

The choice of robotics, as the subject of this study, is not accidental, because it is a promising, high-tech, dynamically developing sphere that is located at the junction of related industries. Many of the world's leading powers are engaged in research and development in this field [4].

\section{Express analysis of the thematic area "Robotics"}

The present study to assess the cyclicality of publications was conducted on the basis of the Web of Science search platform. To analyze publication activity in the WoS system, the following search query ("extended search") was compiled "by category WoS" - WC: "WC = Robotics" with a filter by year "1970-2019."

The search results page presents 168,562 publications and conference materials as of the second quarter of 2019. The first publication in the «Robotics» category appeared in the Web of Science archive in 1989. This year can be considered the beginning of serious publication activity in this area.

We will analyze the current state of development of this area based on data that can be obtained from the abstract database WoS. Figure 1 shows a graph of the total number of publications on a given topic from 1995 to 2018.

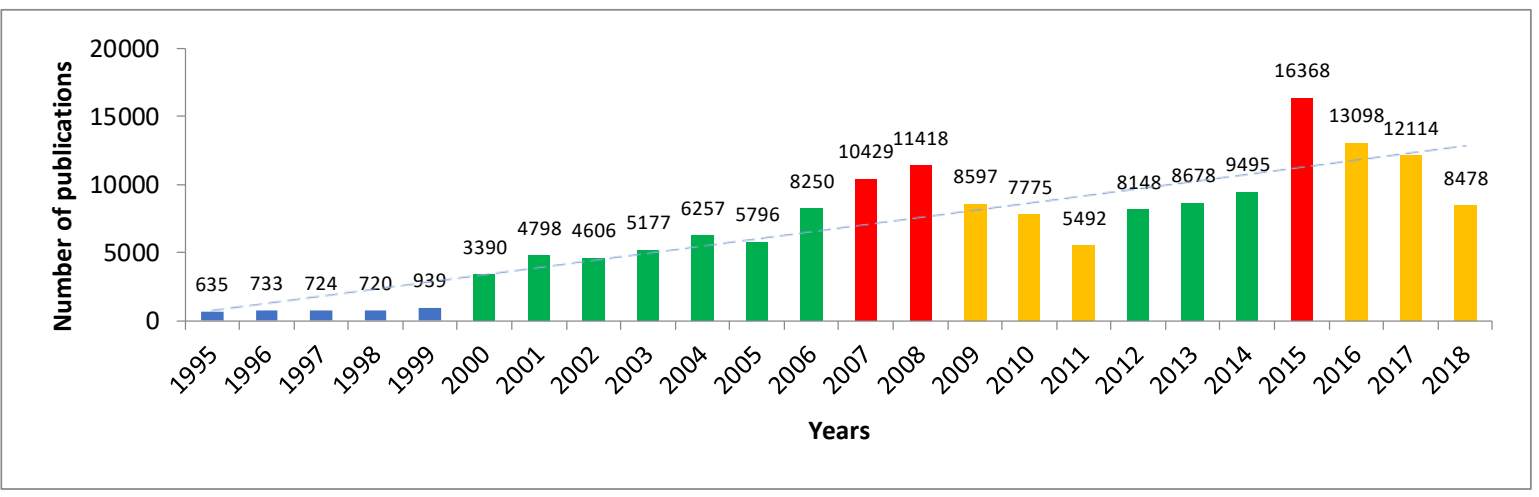

Figure 1. The number of publications on the topic "Robotics" from 1995 to 2018 
Green indicates the years when there is an increasing interest in robotics. The decline in publication activity and the stationary use of technologies are shown in orange. Years of maximum publication activity are highlighted in red. As you can see, from 1989 to 1999. the world community conducted some research and conferences, published articles, but the jump in the development of interest in robotics and the beginning of active publication activities in the world occurred in 19992000. and amounted to almost 2500 publications.

In the period from 2000 to 2008 there was a continuous growth, the latest solutions in technology were introduced. Growth peaked in 2008 at 11418 publications. Further, from 2009 to 2011, some developments were made on the basis of published documents and from 2011 to 2015 there was a new jump in interest and, accordingly, the development of robotics, the number of publications reached almost 7 000. After the peak of growth, as in 2008, publication activity began to decline, which means that since 2015 active development of "tried-and-tested" technologies has been underway.

Figure 2 is a diagram of the relationship of document types published in the archive WoS. The largest number of documents published are conference materials and articles: 127016 documents and 38437 documents, respectively. Consider these directions separately below.

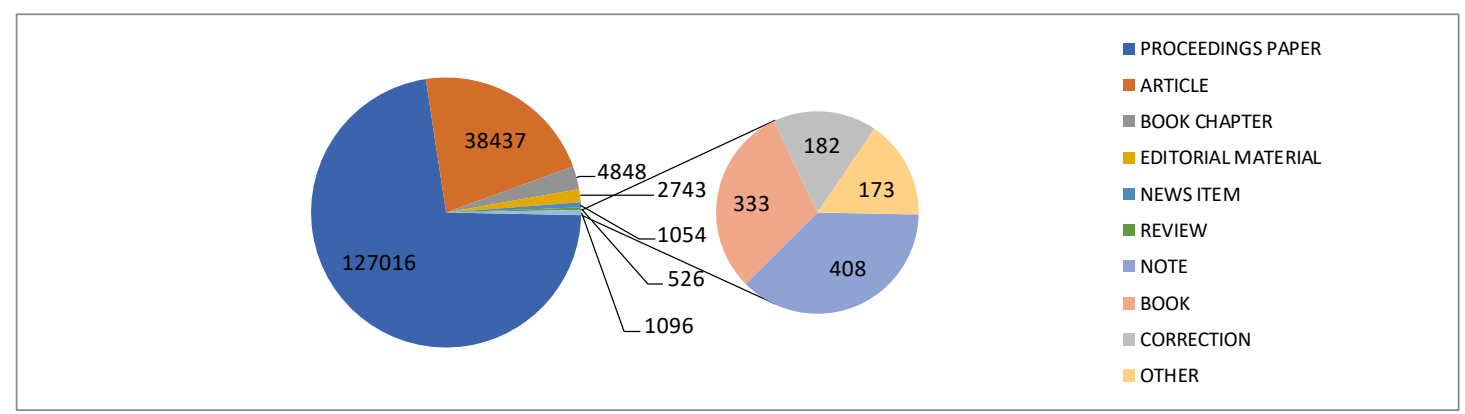

Figure 2. The ratio of the number of all publications on the topic "Robotics"

Figure 3 shows the distribution of the number of documents by country. As you can see in the chart, the United States of America is the leader in the total number of published documents (30 971 documents). They are followed by the People's Republic of China (27 624) and Japan (20 783). It can be concluded that the study of the topic of "Robotics" is most actively engaged in these three countries.

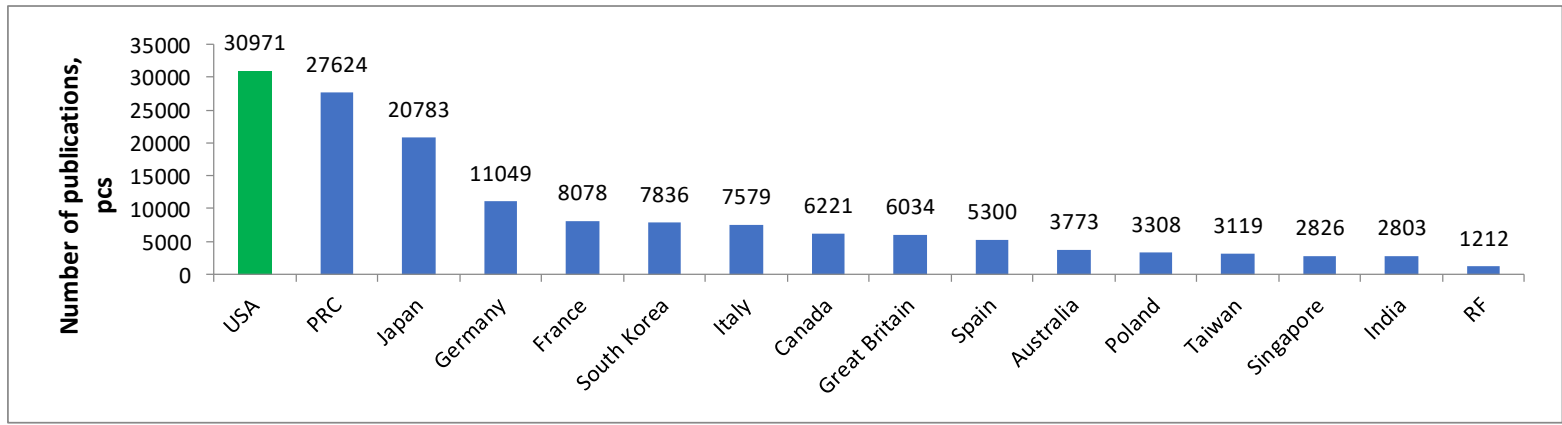

Figure 3. Distribution of publications by country

\subsection{Research of conference materials on the topic "Robotics"}

Let's analyze the current state of documents on thematic conferences based on the data from the WoS abstract database. To do this, we investigate the publication activity by the authors of thematic conferences from 1989 to 2019. Figure 4 presents data on the number of worldwide publications for the specified period. 
From 1989 to March 2019, 127,016 documents of this type were published. According to the diagrams in Figure 4, it can be seen that interest in robotics in the world was manifested in 2000, when the number of publications increased from 95 to 2691. The largest number of documents published after thematic conferences (13 274) is in 2015 year. The smallest number of works (9) is in 1989 and 1991.

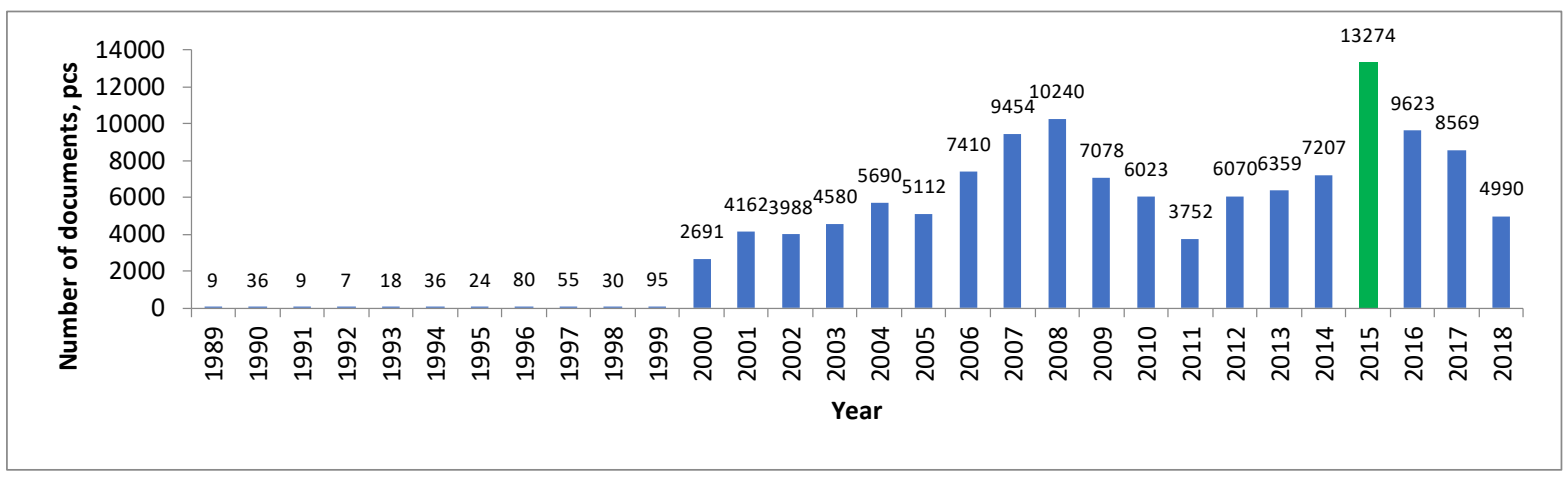

Figure 4. Publications in thematic conferences in 1989-2018

In total, 3725 conferences were held in the direction of Robotics. The leading conferences, at the end of which the largest number of documents are published, are conferences supported by the international non-profit association of specialists in the field of engineering, the Institute of Electrical and Electronics Engineers (IEEE).

\subsection{Research of published articles on the subject of "Robotics"}

Let's analyze the current state of publication of articles on the subject of "Robotics" based on data from the WoS abstract database from 1983 to 2018 [fig. 5]. A total of 38448 documents of this type were published during this period. Based on the data obtained, we can conclude that interest in the topic of "Robotics" is growing from year to year, which is confirmed by the steady increase in the number of published articles. Interestingly, conferences on topics of interest began to be held in 1989 and, accordingly, documents on the results of conferences began to appear at the same time. Articles on the chosen topic began to be published six years earlier, in 1983. The jump in more than 800 published documents is also observed in 2015.

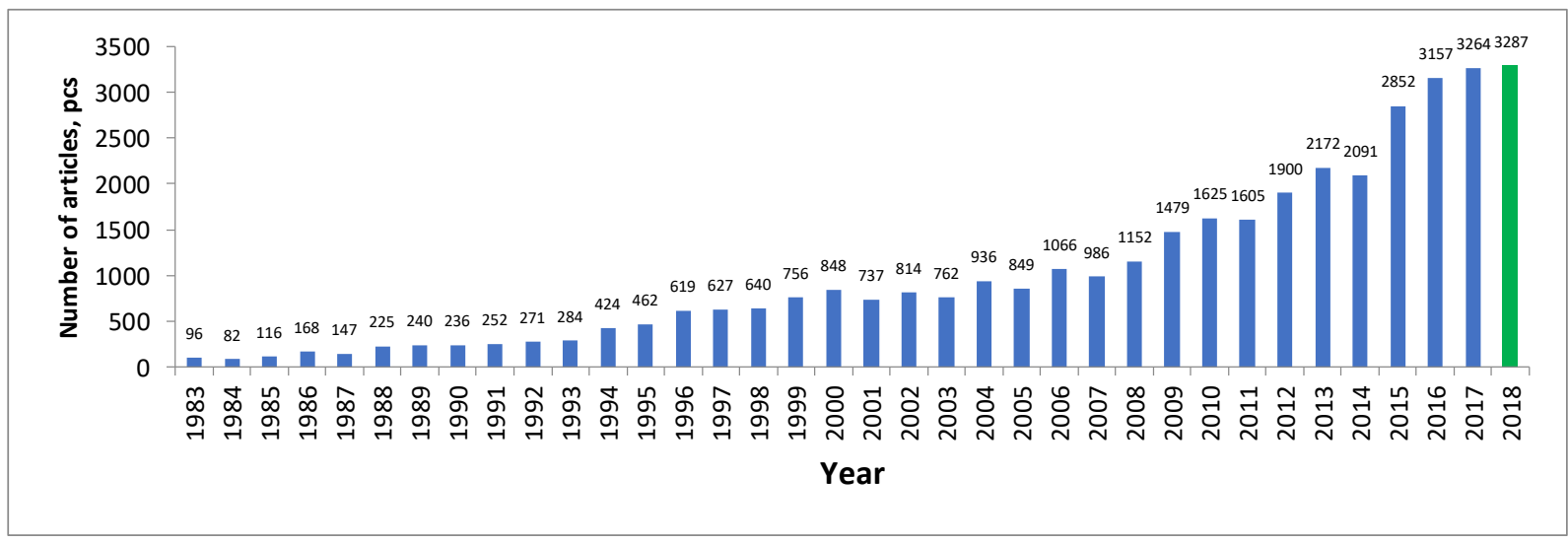

Figure 5. The number of published articles on the subject of "Robotics" from 1983 to 2018

\section{Development of related areas}

The development of robotics is largely determined by the level of development of related industries: the science of materials, as well as the development of computer technology. Data on the conducted interdisciplinary research are presented in Table 1 . 
Table 1. Number of publications in related industries

\begin{tabular}{|l|l|c|}
\hline № & Categories Web of Science & Number of publications \\
\hline 1 & Computer Science, Artificial Intelligence & 75015 \\
\hline 2 & Automated control systems & 69038 \\
\hline 3 & Electrical and Electronic Engineering & 52247 \\
\hline 4 & Computer Science, Cybernetics & 13171 \\
\hline 5 & Computer Science, Theory and Methods & 11224 \\
\hline 6 & Mechanical engineering & 10327 \\
\hline 7 & Computer science, information systems & 9348 \\
\hline 8 & Computer Science, Interdisciplinary Applications & 7985 \\
\hline
\end{tabular}

Based on the data of Table 1, it can be concluded that for the entire time that the Robotics topic exists in the WoS, the largest number of documents affect such related topics as Computer Science, Artificial Intelligence (more than 44\%), Automated Control Systems (more than 41\%) and Electrical and Electronic Engineering (more than 31\%).

In addition to the leading topics (1 300 published documents over 10 years), some areas were selected that are inherently associated with robotics. From the diagram [fig. 6] it can be seen that the jump in the publication of documents precisely on the study of artificial intelligence occurred in 2015. Studies on automation of systems management show a stable number of documents - about 3000 per year. With regard to electrical and electronic engineering, approximately 2500 papers are published annually. Figure 6 also shows the distribution for 6 main headings out of 71 presented. It is in the six areas indicated below that the largest number of documents are published.

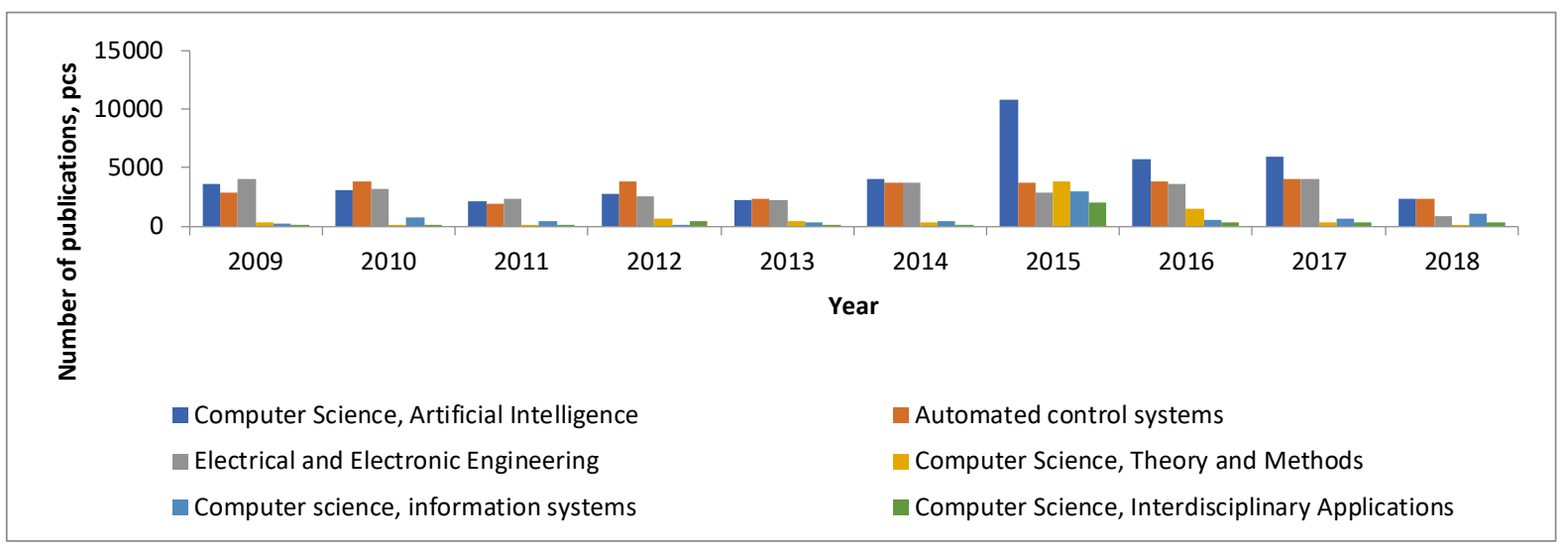

Figure 6. Number of publications on related topics from 2009 to 2018

\section{Conclusions}

In the world, a steady rapid increase in scientific activity was indicated in the 2000s. Leading positions are occupied by the USA, Japan, China.

The development of scientific thought does not occur progressively, but, rather, cyclically. Time is needed for research, development of presented concepts and new results. Such a period can reach 5-7 years, after which a sharp jump in publication activity is visible, then it goes on a decline. More progressive technologies are emerging, scientific thought is developing in accordance with the various needs of time. Past developments are being supplanted and replaced by more efficient nextgeneration technologies. After about 5-7 years, the situation repeats again.

The thematic area "Robotics" is developing intensively, this is evidenced by the active publication activities of leading countries and countries interested in the development and use of robotics. Interest in this area is growing. Due to the fact that robotics is at the junction of industries, such related areas as artificial intelligence, automated control systems, electrical and electronic engineering, and materials science are actively developing in parallel. Therefore, the further development of robotics will largely be determined, including by the development of related areas. 


\section{Acknowledgements}

The study was carried out at the expense of the Russian Science Foundation grant (project \# 19-71-30008).

\section{References}

[1] Sokolov, D.V. Publication Activity as a Scientometric Indicator: Russian and International Experience // The science. Innovation. Education, vol. 9, issue 1, pp. 131-147, 2014.

[2] Piro, Fredrik Niclas, Aksnes, Dag Westreng, Knudsen Christensen Karen, Finnbjørnsson, Thorvaldur, Fröberg, Johan, Gunnarsdottir, Oddny, Karlsson, Staffan, Klausen, Pia Helene, Kronman, Ulf, Leino, Yrjö, Magnusson, Magnus Lyngdal, Miettinen, Maija, Nuutinen, Anu, Poropudas, Olli, Wiborg Schneider, Jesper, Sivertsen, Gunnar. Comparing Research at Nordic Universities using Bibliometric Indicators. Rapport. Nordforsk, № 4, 2011.

[3] Miles, Budimir. Key Trends in Robotics Development // Design World. Available at: https://www.designworldonline.com/key-trends-robotics-development/ (accessed 10.05.2021)

[4] Chambers, Nick. Top Six Trends in Industrial Robotics // Robotics Tomorrow. Available at: https://www.roboticstomorrow.com/article/2014/09/top-six-trends-in-industrialrobotics/4726/ (accessed 10.05.2021) 